\title{
A SÍNDROME DA APNEIA E HIPOPNEIA DO SONO: UMA REVISÃO DE LITERATURA
}

\section{THE OBSTRUCTIVE SLEEP APNEA: A LITERATURE REVIEW}

\author{
Vanda Sanderana Macêdo Carneiro* \\ Maria Helena Chaves de Vasconcelos Catão** \\ Josué Alves"**
}

\begin{abstract}
RESUMO
A Síndrome da Apneia e Hipopneia do Sono (SAHOS) só recentemente foi reconhecida como uma das desordens mundialmente mais prevalentes e subdiagnosticadas, sendo ela a desordem mais comum e responsável pela maior morbidade e mortalidade entre os distúrbios do sono. Os sintomas mais comuns da síndrome são o ronco alto e descontínuo, episódios de ressonares, movimentação brusca do corpo para restabelecer a respiração, sudorese profusa, sonolência diurna excessiva, cansaço crônico e modificações da personalidade com redução da performance motora e intelectual. O diagnóstico da SAHOS é fechado baseando-se no histórico relatado pelo paciente e seu parceiro de sono; no exame de ouvido, nariz e garganta e no exame polissonográfico. As opções de tratamento da SAHOS incluem medidas terapêuticas como a adoção de hábitos para higiene do sono, a cirurgia de uvulopalatofaringoplastia, a cirurgia de avanço mandibular e maxilar, o uso de aparelhos de CPAP e o uso de aparelhos orais de protrusão mandibular (AOPM). Este estudo buscou discutir a relação entre os sinais, sintomas e tratamento do paciente portador da Síndrome da Apneia e Hipopneia do Sono (SAHOS) por meio de revisão de literatura.
\end{abstract}

Descritores: Transtornos do sono

\section{ABSTRACT}

The Obstructive Sleep Apnea (OSA) has only recently been recognized as one of the world's most prevalent underdiagnosed disorders, being the most common disorder and accounts for most morbidity and mortality among sleep disorders. The most common symptoms of the syndrome are loud snoring and discontinuous episodes of snoring, sudden movement of the body to restore breathing, profuse sweating, excessive daytime sleepiness, chronic fatigue and personality changes with reduction of motor and intellectual performance. The diagnosis of OSA is closed based on the history reported by the patient and her partner sleep: the examination of ear, nose and throat, and polysomnography. The options for treatment of OSA include therapeutic measures as adopting habits of sleep hygiene, uvulopalatopharyngoplasty surgery, surgery of maxillary and mandibular advancement, the use of CPAP devices and the use of oral appliances for mandibular protrusion (AOPM). This study sought to discuss the relationship between the signs, symptoms and treatment of patients with Obstructive Sleep Apnea (OSA) by means of literature review.

Descriptors: Sleep Disorders

* Especialista em prótese ASCES; mestranda em clínica odontológica UEPB; vandacarneiro@hotmail.com*

** Dsc. em Laserterapia UFBA; Professora do programa de pós graduação odontologia UEPB; mhelenact@ zipmail.com.br

*** Dsc. em reabilitação oral USP; Professor coordenador especialização prótese ASCES; josuedoca@gmail.com 


\section{N T RO DUÇÃO}

A Síndrome da Apneia e Hipopneia do Sono (SAHOS) vem sendo descrita há mais de 40 anos e só recentemente foi reconhecida como uma das desordens mundialmente mais prevalentes e subdiagnosticadas. É a desordem mais comum observada na prática da medicina do sono, sendo ela responsável pela maior morbidade e mortalidade entre os distúrbios do sono (Levendowski et al. $\left.{ }^{1}, 2007\right)$.

Estima-se que $80 \%$ dos casos de SAHOS são subdiagnosticados, sendo os sintomas mais comuns o ronco alto e descontínuo, episódios de ressonares, movimentação brusca do corpo para restabelecer a respiração, sudorese profusa, sonolência diurna excessiva, cansaço crônico e modificações da personalidade com redução da performance motora e intelectual. A presença hipersonolência diurna representa uma limitação significativa da qualidade de vida do indivíduo, bem como um aumento da morbilidade e mortalidade provinda da SAHOS, visto ser um importante fator de risco para acidentes automobilísticos e laborais, podendo originar alterações cognitivo-comportamentais relevantes (Canani e Barreto ${ }^{2}$, 2001, Mediano et al. $\left.{ }^{3}, 2007\right)$.

O seu diagnóstico definitivo é estabelecido em doentes com sintomas clínicos sugestivos e confirmado por polissonografia (PSG) com demonstração das apneias e hipopneias associadas a perturbações fisiológicas (Ventura et al. ${ }^{4}, 2007$ ).

Entre as opções de tratamento da SAHOS já consagradas na literatura, encontramos como principais terapêuticas as medidas de higiene do sono, a cirurgia de uvulopalatofaringoplastia, a cirurgia de avanço mandibular e maxilar, o uso de aparelhos de CPAP e aparelhos orais de protrusão mandibular (AOPM) (Bahia et al. ${ }^{5}, 2006$, Lorenzi Filho ${ }^{6}, 2001$, George ${ }^{7}$, 2001, Schwarting et al. ${ }^{8}, 2007$, Hoffstein ${ }^{9}$, 2007, Fransson et al. ${ }^{10}, 2003$ ).

Diante do exposto, o objetivo deste estudo é discutir a relação entre os sinais, sintomas e tratamento do paciente portador da Síndrome da Apneia e Hipopneia do Sono (SAHOS) por meio de revisão de literatura.
A evolução do aparelho respiratório humano atribuiu maior relevância respiratória à nossa faringe, transformada em um segmento vulnerável, sem arcabouço e propensa ao colapso durante a inspiração, particularmente durante o sono. Isso tornou possível o surgimento da síndrome da apneia e hipopneia obstrutiva do sono (SAHOS). A atenção médica para o diagnóstico da SAOS e suas consequências tem sido crescente. Com o aumento da expectativa de vida da nossa população, é provável que os impactos mórbidos cumulativos da SAHOS sejam cada vez mais aparentes (Cahali ${ }^{11}$, 2007).

A SAHOS é uma doença crônica, progressiva, incapacitante, com alta mortalidade e morbidade cardiovascular. A prevalência de SAHOS é de $9 \%$ da população masculina de meia idade (30-60 anos) e $4 \%$ da população feminina após a menopausa. Sua prevalência é ainda maior nas faixas etárias mais avançadas. A SAHOS pode ocorrer em qualquer idade, mas acomete principalmente homens obesos entre 40 e 60 anos, sendo menos frequente em mulheres antes da menopausa. Em obesos mórbidos a incidência ultrapassa $50 \%$. O sexo masculino é mais afetado (proporção de 1,4 a 3,6 homens/ mulheres) $-82 \%$ a $95 \%$ dos acometidos são homens de meia idade Reimão e Joo ${ }^{12},(2000)$, devido a diferenças anatômicas das Vias Aéreas Superiores (VAS), perfil hormonal e distribuição adiposa do tipo central nos homens (tronco e pescoço) (Mancini et al. ${ }^{13}, 2000$ ).

Alterações polissonográficas compatíveis com a SAHOS são ainda encontradas em 1 a $2 \%$ das crianças e, dentre os adultos, em $24 \%$ dos homens e $9 \%$ das mulheres. Na faixa etária dos 50 aos 60 anos, tais alterações ocorrem em $31 \%$ dos homens e $16 \%$ das mulheres (Cahali $\left.{ }^{11}, 2007\right)$.

\section{SINAIS E SINTOMAS}

O primeiro sintoma clínico observado é o ronco alto, associado a períodos apneicos de dez segundos ou mais. Outros sintomas comuns são o comportamento anormal durante o sono, movimentação noturna, sonambulismo, cefaleia matinal
CARNEIRO VSM

CATÃO MHCV

ALVES J

A SINNDROME

DA APNEIA E

HIPOPNEIA

DO SONO UMA

REVISÃO DE

LITERATURA

. 135

REV. ODONTOL.

Univ. CID. São

PAULO

2012; 24(2): $134-$

41, MAIO-AGO 
CARNEIRO VSM CATÃO MHCV ALVES J

A SINDROME DA APNEIA E HIPOPNEIA DO SONO UMA REVISÃO DE LITERATURA

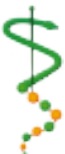

REV, ODONTOL. UNIV, C I D , SÃO PAULO $2012 ; 24(2): 134-$ 41, MAIO-AGO e sonolência diurna. A apneia obstrutiva do sono caracteriza-se pela aposição da língua nas paredes laterais da orofaringe e no palato mole, ocorrendo um colapso destas estruturas, o que leva à diminuição ou à ausência do fluxo aéreo por cerca de 10 segundos, por pelo menos cinco a dez vezes por hora de sono. O indivíduo tenta respirar até a hipoxemia e desperta, em seguida, ocorrendo a desobstrução das vias aéreas e o adormecimento até a repetição do evento. Esse esforço repetitivo para que o ar percorra as vias aéreas causa um aumento de volume de $30 \%$ e a flacidez daquelas estruturas (Ramos e Furquim $^{14}$, 2004).

A obstrução das vias aéreas superiores durante o sono leva à parada da respiração, à diminuição do oxigênio no sangue (por vezes com o aumento do dióxido de carbono) e a um despertar, na maioria das vezes, sem o doente dar por isso (microdespertar), e de novo, pouco depois, reinicia-se todo esse processo. A repetição destes eventos durante a noite leva o doente a queixar-se de diversos sintomas, dos quais se destacam a hipersonia diurna, roncopatia, fadiga, cansaço exagerado ao acordar, paradas respiratórias observadas pelo parceiro(a) de cama, alterações de memória e percepção, disfunções sexuais, enurese noturna, hipertensão arterial (Bahia et al. ${ }^{5}, 2006$ ).

Em crianças, a doença se apresenta geralmente com queixas de ronco, sono inquieto e sensação de sufocação ao sono, sendo particularmente mais comum nos menores de cinco anos de idade. A agitação diurna é frequentemente informada pelos pais e a maioria apresenta obstrução nasal, sendo a rinite alérgica a doença mais freqüentemente citada, seguida da hipertrofia adenotonsilar. O gênero não mostra relevância na prevalência da SAHOS infantil (Ramos et al. ${ }^{15}$, 2006).

A SAHOS acaba por condicionar a presença de hipersonolência diurna, perturbações cardiorrespiratórias e neurocognitivas. Essa síndrome pode apresentar-se aos diferentes clínicos de múltiplas formas, que variam de alterações cardíacas (hipertensão arterial, hipertrofia ventricular esquerda, enfarte do miocárdio, angina noturna, disritmias, insuficiência cardíaca, cor pulmonale), alterações psíquicas (depressão, ansiedade, alterações comportamentais, delírio agudo), alterações neurológicas (epilepsia refratária, acidente vascular cerebral, cefaleias), e alterações urológicas ou gastrenterológicas, entre outras (Ventura et al. $\left.{ }^{4}, 2007\right)$.

A síndrome pode ainda provocar alterações inflamatórias, endoteliais, ateroscleróticas, autonômicas e metabólicas, aumentando o risco para hipertensão arterial sistêmica, arritmias cardíacas, doença nas coronárias e insuficiência cardíaca congestiva. Além disso, o estresse oxidativo resultante da hipoxemia/reoxigenação é associado ao aumento da resistência periférica à ação da insulina, elevação da velocidade de hemossedimentação (VHS) da proteína $\mathrm{C}$ reativa, da interleucina- 6 e do fator de necrose tumoral $\mathbf{\square}$, que são marcadores inflamatórios. Ainda a redução da quantidade de sono profundo (delta) está relacionada à diminuição da secreção do hormônio de crescimento $(\mathrm{GH})\left(\right.$ Cahali $^{11}$, 2007).

Radiograficamente, pode-se observar algumas características anatômicas relevantes no diagnóstico nos pacientes com SAHOS, como: arco mandibular estreito; retrognatia maxilar e mandibular; altura facial inferior aumentada; posição mais baixa e anteriorizada do osso hioide; área faringiana reduzida; ângulo craniocervical aumentado; distância diminuída entre a base da língua e parede posterior da faringe; tonsilas e adenoides hipertrofiadas, dentição maxilar e mandibular sobre-erupcionadas e língua alongada (Almeida et al. $\left.{ }^{16}, 2006\right)$.

Estima-se que $80 \%$ dos casos de SAHOS são subdiagnosticados, sendo também sintomas comuns o cansaço crônico e modificações da personalidade com redução da performance motora e intelectual. A presença de hipersonolência diurna representa uma limitação significativa da qualidade de vida do indivíduo, bem como um aumento da morbilidade e mortalidade da SAHOS, visto ser um importante fator de risco para acidentes automobilísticos e laborais, podendo originar alterações cognitivo-comportamentais relevantes (Canani e Barreto ${ }^{2}$, 2001, Mediano et al. $\left.{ }^{3}, 2007\right)$. 
Nesse grupo de pacientes atribui-se a dificuldade de manter-se alerta ao risco aumentado de acidentes automobilísticos. Pacientes com dez ou mais eventos obstrutivos por hora de sono têm risco 6,3 maior que indivíduos com sono normal, mostrando forte associação entre apneia do sono e o risco para acidentes automobilísticos (IC 95\%) (Canani e Barreto $^{2}$, 2001). O relato de mortalidade relacionada à apneia obstrutiva do sono tem aumentado significativamente, quando o número de apneias passa de 20 por hora de sono (Almeida et al. ${ }^{16}, 2006$ ).

O seu diagnóstico definitivo é estabelecido em doentes com sintomas clínicos sugestivos e confirmado por polissonografia (PSG) com demonstração das apneias e hipopneias associadas a perturbações fisiológicas(Ventura et al. ${ }^{4}$, 2007). O diagnóstico da SAHOS baseia-se, então, no histórico relatado pelo paciente e seu parceiro de sono; no exame de ouvido, nariz e garganta; no índice de massa corporal (IMC); e no exame polissonográfico, permitindo a distinção entre a síndrome e um simples ronco (Nayar e Knox ${ }^{17}$, 2005).

A polissonografia é um exame fundamental na avaliação de pacientes; primeiro, por estabelecer critérios de gravidade da síndrome; segundo, devido à alta taxa de indivíduos assintomáticos com índices polissonográficos alterados nos obesos, em especial nos obesos graves (Gregório et al. $\left.{ }^{18}, 2007\right)$.

Por ser um exame de alta complexidade, a polissonografia só é realizada em grupos de risco ou suspeitos. Ela consiste na monitoração, durante uma noite inteira, de diversos parâmetros fisiológicos, incluindo o eletroencefalograma, eletrocardiograma, eletroculograma, eletromiograma, medida de fluxo aéreo bucal e nasal, esforço respiratório por meio de pneumógrafo e medida de saturação transcutânea contínua de O2 por meio de oxímetro. Ela caracteriza nos pacientes apneicos o índice de apneia-hipopneia (IAH), a dessaturação da Oxi-hemoglobina, os microdespertares, as porcentagens dos estágios, o ECG, o registro do Ronco e da posição corporal(Reimão e JoO $^{12}$, 2000).
$\mathrm{O}$ índice de apneias + hipopneias por hora de sono (IAH), o qual é também denominado RDI (respiratory disturbance índex, ou índice de distúrbios respiratórios) classifica o grau da apneia, e sua quantificação se dá em episódios por hora de sono. A SAHOS é classificada como leve quando o IAH encontra-se entre 5 e 15 , o quadro é dito moderado quando o índice apresenta-se entre 15 e 30, e acentuado quando esse índice é maior que 30 (Tangerina et al. ${ }^{19}$, 2008).

A oximetria da polissonografia é usada para caracterizar a frequência e profundidade da dessaturação do oxigênio (O2). Na medicina do sono, ela tem sido um instrumento essencial que permite detectar as rápidas flutuações na saturação arterial de oxigênio durante o sono, uma característica dos doentes com apneia do sono, permitindo a detecção precoce dos distúrbios respiratórios. Tem sido debatida, na literatura, não só a utilidade da oximetria no screening de doentes com distúrbios de sono, mas até a possibilidade de substituir a PSG nalgumas circunstâncias. Entretanto, não há estudos para validação da utilização isolada da oximetria noturna na detecção de distúrbios respiratórios do sono (Ventura et al. ${ }^{4}, 2007$ ).

Há, ainda, relação da diminuição da oxigenação durante o sono ao aumento de prevalência de pesadelos. Estudando a frequência de pesadelos em pacientes com SAHOS e a relação dos mesmos com os parâmetros respiratórios, se observou que, apesar de alguns exemplos ilustrativos de correlação entre desaturação de oxigênio e ocorrência de sonhos, os parâmetros respiratórios que mensuram a severidade da síndrome não se correlacionaram substancialmente com a frequência de pesadelos (Schredl et al. ${ }^{20}, 2006$ ).

Entre as opções de tratamento da SAHOS encontram-se como principais terapêuticas a cirurgia de uvulopalatofaringoplastia, a cirurgia de avanço mandibular e maxilar, o uso de aparelhos de CPAP e aparelhos orais de protrusão mandibular (AOPM). O Continuous Positive Air Pressure (CPAP) é o aparelho indicado quando o quadro da apneia apresenta-se mais evoluído, precisando de altos ní-
CARNEIRO VSM CATÃO MHCV ALVES J

A SINNDROME

DA APNEIA E HIPOPNEIA DO SONO UMA REVISÃO DE LITERATURA

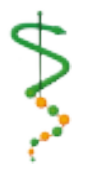

REV. ODONTOL. Univ. CID. São PAULO 2012; 24(2): $134-$ 41 , MAIO-AGO 
CARNEIRO VSM CATÃO MHCV ALVES J

A SÍNDROME DA APNEIA E HIPOPNEIA DO SONO UMA REVISÃO DE LITERATURA

\section{8}

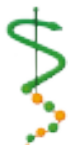

REV, ODONTOL. UNIV. CID, SÃO PAULO $2012 ; 24(2): 134-$ 41, MAIO-AGO veis de pressão para ventilação, onde ele funciona como uma tala pneumática que mantém as vias aéreas superiores abertas durante o sono. O uso deve ser durante todas as noites, e nova avaliação deve ser feita para determinar se a pressão deve ser reajustada ou o tratamento descontinuado (Lorenzi Filho ${ }^{6}, 2001$ ).

Os aparelhos de protrusão mandibular vêm sendo crescentemente reconhecidos como um tratamento alternativo ao CPAP. Comparações entre o CPAP e o AOPM revelaram que, apesar de o aparelho ser menos eficiente na redução do índice de distúrbios respiratórios, eles são, em média, utilizados com maior frequência, sendo preferidos por mais pacientes e mais prontamente aceitos que o CPAP (Levendowski et al. $\left.{ }^{1}, 2007\right)$. Os AOPMs são uma boa alternativa para o tratamento do ronco e da SAHOS devido ao seu custo reduzido e ao relativo conforto de uso, tendo uma maior aceitação por parte dos pacientes. Ainda há dúvidas sobre a eficácia dos apareIhos intrabucais no tratamento do ronco e SAHOS, mas seu uso no tratamento da apneia de gravidade média tem recebido grande atenção e aceitabilidade(Almeida et al. $\left.{ }^{16}, 2006\right)$.

Quanto à indicação dos AOPM, não existe ainda um consenso. No uso em pacientes com SAHOS, observou-se redução dos índices de apneia-hipopneia, redução do ronco e melhora nas atividades diárias. O aparelho oral reduziu o índice de apneia e hipopneia (IAH) para menor que 10 em $54 \%$ dos casos e reduziu o ronco em 45\%(Hoffstein $\left.{ }^{9}, 2007\right)$.

Os aparelhos podem ser classificados em ajustáveis ou em monobloco. $\mathrm{O}$ número de pesquisas realizadas é insuficiente para avaliar os prós e contras do ajuste em contraposição à estabilidade do aparelho em monobloco. Elas não trazem evidências suficientes para mostrar que os aparelhos ajustáveis são mais eficazes ou mais confortáveis de usar que os apareIhos em monobloco (George ${ }^{7}, 2001$ ).

Os aparelhos ajustáveis são confeccionados em laboratório protético com splintagem ajustável para adaptar a mandíbula à maxila do paciente. Essa splintagem posiciona a mandíbula, a língua e estruturas adicionais mais anteriormente, e ainda au- mentando a dimensão vertical de oclusão, resultando numa abertura do lúmen da faringe, o que reduz a resistência nessa via aérea e a mantém aberta mecanicamente durante o sono (Schwarting et al. ${ }^{8}, 2007$ ).

É importante ressaltar ainda que há outras medidas para o tratamento da SAHOS, como as medidas de higiene do sono (MHS). Avaliando-se o impacto de um folheto sobre MHS em 36 doentes recém-diagnosticados com SAHOS, esperava-se que a informação sobre as MHS transmitida alterasse hábitos do sono, e que a intervenção do folheto informativo se traduzisse numa melhoria da avaliação subjetiva das queixas relativas ao sono. Foram avaliados os hábitos e as queixas relativas ao sono antes e após intervenção, e observou-se que não houve diferenças significativas na adoção de MHS entre os dois momentos, não sendo a mudança evidente devido o fato dos doentes já possuírem uma higiene do sono razoável no pré-teste, e também pelo fato da escala utilizada não ser suficientemente discriminativa. Além disso, a exigência de uma autodisciplina pode ter coincidido com exigências como a adaptação ao tratamento com CPAP (Bahia et al. ${ }^{5}, 2006$ ).

\section{I SCUSSÃO}

Quando da prevalência da SAHOS comparada entre os sexos, os resultados obtidos nos estudos revisados mostraram sempre uma maior prevalência em indivíduos do sexo masculino (Canani e Barreto $^{2}, 2001$, Cahali ${ }^{11}, 2007$, Reimão e Joo ${ }^{12}$, 2000 Mancini et al. ${ }^{13}$, 2000, Ramos e Furquim $^{14}$, 2004). Essa maior prevalência no sexo masculino provavelmente se deve à disposição adiposa do tipo central de gordura no corpo dos homens, além de diferenças anatômicas das Vias Aéreas Superiores (VAS) e do perfil hormonal do sexo masculino (Mancini et al. ${ }^{13}, 2000$ ).

Com relação à faixa etária mais prevalente apresentada pelos estudos, fora descrito que a doença é mais prevalente entre indivíduos com idade entre 40 e 60 anos. A literatura também relaciona a severidade da síndrome à idade do paciente, sendo observado que pacientes mais idosos têm doença mais grave, enfatizando-se o caráter progressivo da doença (Mancini et 
al. $^{13}$, 2000, Ramos e Furquim ${ }^{14}$, 2004). A fisiopatologia da SAHOS tem estreita relação com a obesidade, de forma que $60 \%$ a 90\% dos indivíduos com SAHOS têm índice de massa corpórea (IMC > $29 \mathrm{~kg} / \mathrm{m} 2$ ), mas ainda não foi estabelecida uma relação progressiva entre as variáveis (Lorenzi Filho $^{6}, 2001$, Hoffstein ${ }^{9}$, 2007, Almeida et al. $\left.{ }^{16}, 2006\right)$.

Dentre as opções de tratamento para distúrbios do sono, encontra-se como principais terapêuticas empregadas a adoção de medidas de higiene do sono, a cirurgia de uvulopalatofaringoplastia, a cirurgia de avanço mandibular e maxilar, o uso de aparelhos de CPAP e aparelhos orais de protrusão mandibular (AOPM) (Bahia et al. ${ }^{5}, 2006$, Lorenzi Filho ${ }^{6}, 2001$, George $^{7}, 2001$, Schwarting et al. ${ }^{8}, 2007$, Hoffstein ${ }^{9}$, 2007, Fransson et al. $\left.{ }^{10}, 2003\right)$. A terapia com a pressão positiva contínua nas vias aéreas (CPAP - continuous positive airway pressure) minimiza o risco de complicações respiratórias e cardiovasculares relacionadas à SAHOS. O nível de ventilação do CPAP necessário é muito variável e deve ser individualizado (Tangerina et al. $\left.{ }^{19}, 2008\right)$. O uso de CPAP é um tratamento eficaz, mas deve-se ter em mente que ele, ao não curar o distúrbio ventilatório, deve ser usado durante toda a vida (Borges e Paschoal21, 2005). Entre suas desvantagens, encontram-se o alto custo e o desconforto, que muitas vezes fazem com que o paciente não tenha a adesão adequada à terapia (Hoffstein ${ }^{9}$, 2007, Almeida et al. ${ }^{16}$, 2006).

A atuação do cirurgião-dentista vem se tornando cada vez mais expressiva no tratamento desse distúrbio do sono com aparelhos orais. Um exemplo disto é que comparações entre o CPAP e o AOPM revelaram que, apesar de este aparelho ser menos eficiente na redução do índice de distúrbios respiratórios, ele é, em média, utilizado com maior frequência, sendo preferido por mais pacientes e mais prontamente aceito que o CPAP, quer seja pelo conforto, quer seja pelo custo (Levendowski et al. ${ }^{1}, 2007$, Almeida et al. ${ }^{16}$, 2006).

Avaliando o desconforto e mensurações sonoras para o ronco de pacientes depois do uso do aparelho de protrusão mandibular por dois anos, 90\% dos pa- cientes notaram uma redução do ronco e apnea, $76 \%$ obtiveram uma redução do cansaço diurno, e $84 \%$ observaram meIhora na qualidade do sono noturno, apresentando este último índice uma melhora de $50 \%$ do quadro inicial dos pacientes (Fransson et al. ${ }^{10}, 2003$ ).

Quanto ao emprego dos aparelhos orais no tratamento de distúrbios do sono, observou-se que sua indicação se dá principalmente nos casos de baixa e média gravidade(Almeida et al. $\left.{ }^{16}, 2006\right)$. Já quanto ao tipo de aparelho - se deve ser utilizado o aparelho ajustável com splintagem ou em monobloco - que deve ser empregado para cada caso, não há um consenso. No uso em pacientes com SAHOS, observou-se redução dos índices de apneia-hipopneia, redução do ronco e melhora nas atividades diárias. O apareIho oral reduziu o índice de apneia e hipopneia (IAH) para menor que 10 em 54\% dos casos e reduziu o ronco em $45 \%$. Em comparação com outros tratamentos, o aparelho apresentou redução de $42 \%$ do IAH contra $70 \%$ do CPAP e $30 \%$ da uvulolaringoplastia; o diferencial consta no fato dos pacientes preferirem usar o AOPM quando comparado ao CPAP. Em 30 meses, $56-68 \%$ dos pacientes continuam a usar o aparelho oral(Hoffstein $\left.{ }^{9}, 2007\right)$.

Muitos pacientes sofrem de distúrbios das vias aéreas superiores que afetam a qualidade do seu sono e, apesar do profissional poder atuar com sucesso no tratamento dessas desordens, poucos o fazem. Essa falta de participação muito ocorre em reflexo à deficiente ênfase dada pela educação nas faculdades a essa área de estudo como parte do seu currículo (Ivanhoe et al..22, 2003). O Cirurgião-dentista precisa mudar sua concepção e torná-la mais ampla, passando a desempenhar um papel ativo no tratamento dessas desordens que, na realidade clínica, são subdiagnosticadas e que, quando da aplicabilidade da sua sintomatologia epidemiologicamente, vai se apresentar através de números importantes em acidentes automobilísticos e laborais.

\section{CONCLUSÃO}

Diante dos dados encontrados na literatura, pode-se observar o paciente portador
CARNEIRO VSM CATÃO MHCV ALVES J

A SINNDROME

DA APNEIA E HIPOPNEIA DO SONO UMA REVISÃO DE LITERATURA

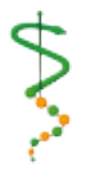

REV. ODONTOL. UNIV, CID . SÃO PAULO $2012 ; 24(2): 134-$ 41, MAI $0-A G 0$ 
CARNEIRO VSM CATÃO MHCV ALVES J A SÍNDROME DA APNEIA E HIPOPNEIA DO SONO UMA REVISÃO DE LITERATURA

REV. ODONTOL

UNIV, CID, SÃO PAULO $2012 ; 24(2): 134-$ 41, MAIO-AGO: de SAHOS apresenta um quadro clínico que pode ser tratado com a confecção de aparelhos orais na clínica odontológica, propiciando um maior bem-estar físico e mental através de terapêutica de conforto e baixo custo.

\section{REFERÊNCIAS}

1. Levendowski DJ, Morgan TD, Patrickus JE, Westbrook PR, Berka C, Zavora T, et al. In-home evaluation of efficacy and titration of a mandibular advancement device for obstructive sleep apnea. Sleep Breath 2007 Sep;11(3):139-47.

2. Canani SF, Barreto SSM. Sonolência e acidentes automobilísticos. J Pneumol 2001 mar./abr.;27(2):94-6.

3. Mediano O, Barceló A, Peña M, Gozal D, Agusti A, Barbé F. Hipersonolência diurna e variáveis polissonográficas em doentes com síndroma de apneia do sono. Rev Port Pneumol 2007 dez.;13(6):896-8.

4. Ventura C, Oliveira AS, Dias R, Teixeira J, Canhão C, Santos O, et al. Papel da oximetria nocturna no rastreio da síndroma de apneia-hipopneia obstrutiva do sono. Rev Port Pneumol 2007 jul.;13(4):525-51.

5. Bahia MG, Soares V, Winck JC. Impacto da higiene do sono em doentes com síndroma de apneia obstrutiva do sono. Rev Port Pneumol 2006 mar;12(2):147-76.

6. Lorenzi Filho G. Como deve ser tratado um paciente com obesidade mórbida e apnéia do sono? Rev Assoc Méd Bras 2001 jul./set.;47(3):177-.

7. George PT. Is adjustability advantageous in mandibular advancement appliances in the treatment of sleep-disordered breathing? Sleep Breath 2001 Sep;5(3):139-47.

8. Schwarting S, Huebers U, Heise M, Schlieper J, Hauschild A. Position paper on the use of mandibular advancement devices in adults with sleep-related breathing disorders. A position paper of the German Society of Dental Sleep Medicine (Deutsche Gesellschaft Zahnaerztliche Schlafmedizin, DGZS). Sleep Breath 2007 Jun;11(2):125-6.

9. Hoffstein V. Review of oral appliances for treatment of sleep-disordered breathing. Sleep Breath 2007 Mar;11(1):1-22.

10. Fransson AM, Tegelberg A, Leissner L, Wenneberg B, Isacsson G. Effects of a mandibular protruding device on the sleep of patients with obstructive sleep apnea and snoring problems: a 2-year follow-up. Sleep Breath 2003 Sep;7(3):131-41.

11. Cahali MB. Conseqüências da síndrome da apnéia obstrutiva do sono. Rev Bras Otorrinolaringol 2007 maio./ju.;73(3):290-.

12. Reimão R, Joo SH. Mortalidade da apnéia obstrutiva do sono. Rev Assoc Méd Bras 2000 jan./mar.;46(1):52-6.

13. Mancini MC, Aloe F, Tavares S. Apnéia do sono em obesos. Arq Bras Endocrinol Metab 2000 fev.;44(1):81-90.

14. Ramos L, Furquim L. Aparelho para apnéia obstrutiva do sono. R Clín Ortodon Dental Press 2004 abr./maio;3(2):21-6.

15. Ramos RTT, Daltro CHdC, Gregório PB, Souza LSdF, Andrade NAd, Andrade Filho AdS, et al. SAHOS em crianças: perfil clínico e respiratório polissonográfico. Rev Bras Otorrinolaringol 2006 maio/jun.;72(3):355-61.

16. Almeida MAO, Teixeira AOB, Vieira LS, Quintão CCA. Tratamento da síndrome da apnéia e hipopnéia obstrutiva do sono com aparelhos intrabucais. Rev Bras Otorrinolaringol 2006 set./out.;72(5):699-703. 
17. Nayar S, Knox J. Management of obstructive sleep apnea in an edentulous patient with a mandibular advancement splint: a clinical report. J Prosthet Dent 2005 Aug;94(2):108-11.

18. Gregório PB, Athanazio RA, Bitencourt AGV, Neves FBCS, Daltro C, Alves E, et al. Apresentação clínica de pacientes obesos com diagnóstico polissonográfico de apnéia obstrutiva do sono. Arq Bras Endocrinol Metab 2007 out.;51(7):1064-8.

19. Tangerina RP, Martinho FL, Togeiro SM, Gregório LC, Tufik S, Bittencourt LR. Achados clínicos e polissonográficos em pacientes com obesidade classe III. Rev Bras Otorrinolaringol 2008 jul./ago.;74(4):579-82.

20. Schredl M, Schmitt J, Hein G, Schmoll T, Eller S, Haaf J. Nightmares and oxygen desaturations: is sleep apnea related to heightened nightmare frequency? Sleep Breath 2006 Dec;10(4):203-9.

21. Borges PTM, Paschoal JR. Indicação inicial de tratamento em 60 pacientes com distúrbios ventilatórios obstrutivos do sono. Rev Bras Otorrinolaringol 2005 nov./ dez.;71(6):740-6.

22. Ivanhoe JR, Frazier KB, Parr GR, Haywood VB. The teaching and treatment of upper airway sleep disorders in North American dental schools. J Prosthet Dent 2003 Mar;89(3):292-6.

Recebido em: 25/04/2011

Aceito em: 15/09/2011

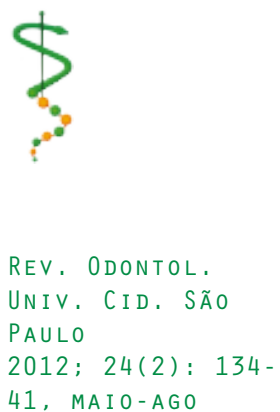

